\title{
High seroprevalence of feline morbilliviruses in free-roaming domestic cats in Chile
}

\author{
Johannes Busch ${ }^{1} \cdot$ Irene Sacristán ${ }^{2} \cdot$ Aitor Cevidanes $^{2}$ - Javier Millán ${ }^{3,4,5}$ - Thomas W. Vahlenkamp ${ }^{1}$. \\ Constanza Napolitano $0^{6,7} \cdot$ Michael Sieg $^{1}$ (i)
}

Received: 15 July 2020 / Accepted: 30 September 2020 / Published online: 20 November 2020

(c) The Author(s) 2020

\begin{abstract}
Feline morbillivirus infections have gained increased attention due to repeated reports of their association with urinary tract disease in cats. In the present study, 112 serum samples from free-roaming domestic cats in Chile were tested for antibodies against feline morbillivirus genotypes 1 and 2 (FeMV-1 and FeMV-2) using an indirect immunofluorescence assay. In total, $63 \%$ of the animals showed antibodies against one or both FeMV genotypes. Antibodies directed exclusively against FeMV-2 were significantly more prevalent in male cats. The correlation of sex and FeMV-2 infection might give insight into potential routes of transmission. We provide, for the first time, serological data on FeMV in Chile.
\end{abstract}

The family Paramyxoviridae currently comprises 78 virus species divided into four subfamilies and 17 genera covering a broad host range including mammals, birds, fish and reptiles [1]. In 2012, a new paramyxovirus was detected in stray cats from Hong Kong, designated as feline morbillivirus (FeMV, formally known as FmoPV) [2]. Subsequent studies verified FeMV to be present in Japan [3], Germany [4], Italy [5], the USA [6], Brazil [7], Turkey [8], the UK [9], Malaysia [10] and mainland China [11]. In-depth analysis of complete genome sequences revealed viral diversity of FeMV strains from different locations [3, 12, 13]. In 2019, a large surveillance program in Germany identified a second genotype of FeMV (FeMV-2) with 78\% whole-genome

Handling Editor: Bert K. Rima.

Electronic supplementary material The online version of this article (https://doi.org/10.1007/s00705-020-04882-2) contains supplementary material, which is available to authorized users.

Constanza Napolitano

constanza.napolitano@ulagos.cl

$\triangle$ Michael Sieg

michael.sieg@vetmed.uni-leipzig.de

1 Institute of Virology, Faculty of Veterinary Medicine, Leipzig University, An den Tierkliniken 29, 04103 Leipzig, Germany

2 PhD Program in Conservation Medicine, Facultad de Ciencias de la Vida, Universidad Andres Bello, República 252, Santiago, Chile nucleotide sequence identity to the previously detected FeMV isolates [14]. In infected cats, viral proteins were predominantly detected in the kidney but were also found in other organs (e.g., lymph nodes) [2, 12, 13, 15]. FeMV involvement in chronic kidney diseases (CKD) has been suggested. CKD is common in domestic cats, with a reported incidence of $28-50 \%$, primarily affecting older animals [16, 17]. Prevalence data for FeMV obtained by detection of antibodies against the viral nucleoprotein $(\mathrm{N})$ in Japan, Hong Kong and the UK revealed that 21.0, 27.8 and $30 \%$ of the animals, respectively, were FeMV positive, [2, 9, 12]. Similar results were obtained using a phosphoprotein (P)-based enzyme-linked immunosorbent assay (ELISA) [18]. In the USA and Brazil, FeMV has been detected by RT-PCR [6, 7], but so far, no serological studies have been published.

We analyzed serum samples from 112 domestic cats. The cohort comprised 62 female and 50 male, rural, free-roaming, mix-bred, short-haired cats, with an average age of 30

3 Facultad de Ciencias de la Vida, Universidad Andres Bello, República 252, Santiago, Chile

4 Instituto Agroalimentario de Aragón-IA2 (Universidad de Zaragoza-CITA), Miguel Servet 177, 50013 Zaragoza, Spain

5 Fundación ARAID, Avda. de Ranillas, 50018 Zaragoza, Spain

6 Departamento de Ciencias Biológicas y Biodiversidad, Universidad de Los Lagos, Av. Fuchslocher 1305, Osorno, Chile

7 Instituto de Ecología y Biodiversidad (IEB), Santiago, Chile 
\pm 4.2 months. Over $90 \%$ of the cats were less than 4 years of age. None of the animals had been neutered, vaccinated or dewormed. The sampling area comprised nine regions from central to southern Chile. In the present study, we determined the antibody status against FeMV-1 and FeMV-2 using an immunofluorescence assay (IFA) developed for both genotypes. Viruses were isolated from urine samples taken from two persistently infected cats in Germany. Propagation in cell culture was performed as described previously for FeMV-1 [19] and for FeMV-2 [14]. Whole genome sequences are available under GenBank accession no. MG563820 and MK182089, respectively. In brief, CrFK cells were infected with FeMV-1 $(\mathrm{MOI}=0.01)$, and LLCMK2 cells were infected with FeMV-2 $(\mathrm{MOI}=0.01)$. After five days, cells were fixed with $80 \%$ acetone, blocked with $5 \%(\mathrm{w} / \mathrm{v}) \mathrm{BSA}$ in PBS before cat sera were applied at a dilution of $1 / 100(\mathrm{v} / \mathrm{v})$ in $1 \%(\mathrm{w} / \mathrm{v})$ BSA in PBS overnight at $4^{\circ} \mathrm{C}$. After washing with PBS, a goat anti-cat $\operatorname{IgG}(\mathrm{H}+\mathrm{L})$ Alexa Fluor 488-conjugated antibody (Dianova, Germany), diluted 1/500 (v/v) in 1\% (w/v) BSA in PBS was applied for $30 \mathrm{~min}$ at $37^{\circ} \mathrm{C}$. Prior to evaluation of the signal, cells were washed twice with PBS. Uninfected cells served as negative controls for each sample and allowed determination of virus-specific signals. Graphs as well as figures were generated using MS Office, and statistics were performed using the GraphPad QuickCalcs website to determine significance by two-tailed Fisher's exact test [20]. RT-PCR for the detection and further phylogenetic analysis of feline morbilliviruses was not possible due to the limited amount of serum available.

The IFA approach was evaluated using sera from the same persistently infected cats whose urine had been used to propagate the respective FeMV genotype, as well as specific antibodies against the FeMV N or P protein (shown in the supplementary file). Based on the observed fluorescence signals, cat sera were judged to be positive for FeMV-1 only, FeMV-2 only, positive for both genotypes (FeMV double positive) or FeMV negative. Representative results are shown in Figure 1, which shows viral intracytoplasmic inclusion bodies indicated by arrows. We found that $63 \%$ of the cats (71 animals) had antibodies against FeMV. Thirty percent of these samples were seropositive for both genotypes. It is currently unknown whether double-positive sera are the result of coinfections or consecutive infections, or possibly due to cross-reactive antibodies derived from either FeMV-1 or FeMV-2 monoinfection. Furthermore, $24 \%$ and $9 \%$ of
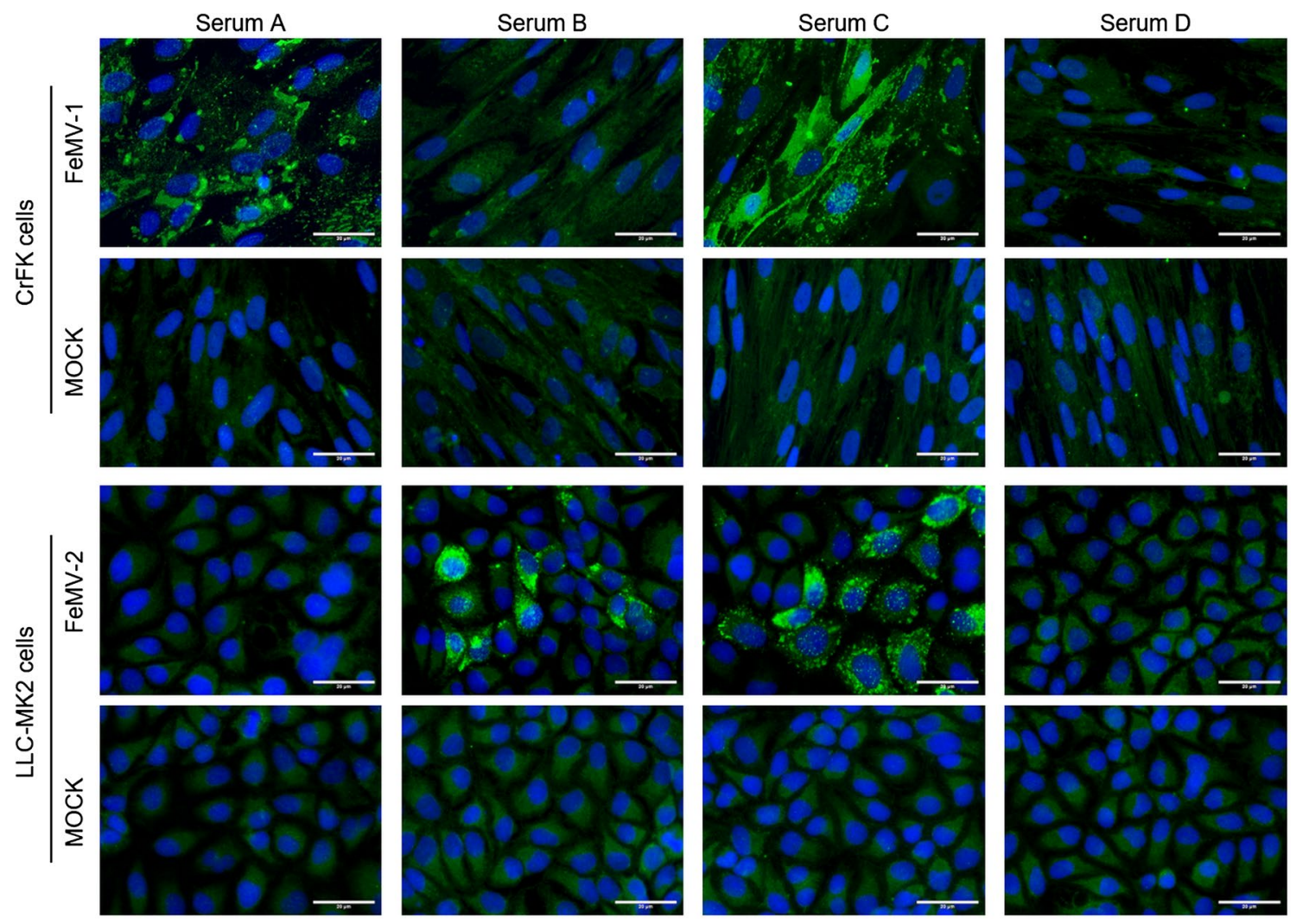

Fig. 1 Representative images of IFA against both FeMV genotypes. Serum A was found to be positive for FeMV-1 only, serum B was positive for FeMV-2 only, serum $\mathrm{C}$ was positive for both types, and serum D was negative for both types. Arrows indicate virus-specific signals. Scale bars indicate $20 \mu \mathrm{m}$. 
the animals were positive FeMV-1 only and FeMV-2 only, respectively (Fig. 2). In combination with the staining pattern observed in Supplementary Figure 1, these data suggest that sera that are positive against only one genotype might be restricted in their response to either one viral protein or even a specific epitope. In addition, sex-related differences in seroprevalence of FeMV were investigated. As depicted in Figure 3, only slight differences between female (69\%) and male (74\%) cats were observed in the overall FeMV seroprevalence. However, statistically significant differences were detected between the sexes for the seroprevalence of FeMV-2 only ( $p=0.0407)$. While $16 \%$ of male cats had antibodies against FeMV-2 only, just 3\% of the female cats were seropositive for FeMV-2 only. Such a correlation was not observed for FeMV-1.

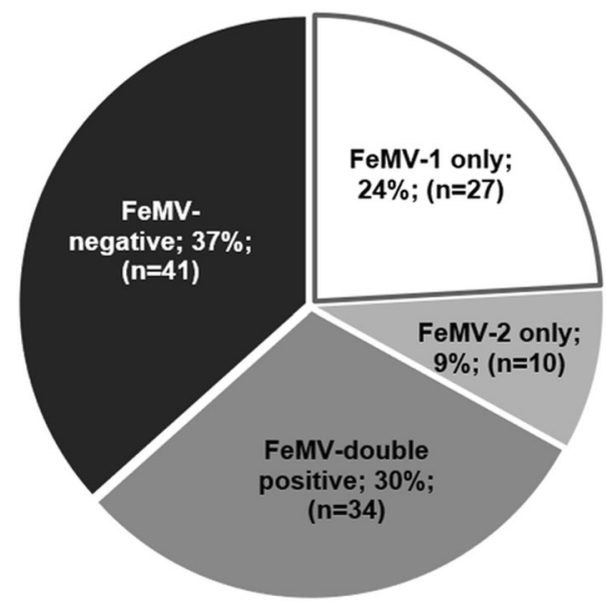

Fig. 2 Seroprevalence of FeMV antibodies determined in samples from free-roaming domestic cats in Chile. Sera were tested separately for antibodies against each genotype
Overall, our results are in accordance with previous studies using recombinant viral $\mathrm{N}$ protein in immunoblot assays [2, 9], N-protein-expressing cells [12], or recombinant-P-protein-based ELISA [18]. Our experimental setting allowed the detection of antibodies against all viral structural proteins simultaneously. This advantage might explain the higher seroprevalence detected in Chilean cats compared with studies from other countries. For instance, experiments conducted in Hong Kong, Japan, and the UK [2, $9,12]$, used single-protein-based assays and thus might be unable to detect antibody responses to other viral proteins, e.g., the viral surface proteins. This is supported by a study using whole-virus immunoblot analysis [3], which confirmed differences in antibody reactivity against structural FeMV proteins. The FeMV-specific antibody prevalence in Japanese cats was found to be $22 \%$. Differences compared to our data (24\% FeMV-1 only and additionally $30 \%$ FeMV double positive) might be due to the limited sample size $(n=13)$ in the previous study or country-specific differences in the epidemiological situation. Samples included in the study by Sakaguchi et al. published in 2014 were obtained from cats brought to a veterinary clinic for various reasons. Those cats are thus likely to have had an owner. In comparison, the cats in Chile analyzed in this study had an owner but were not confined and were in a rural setting (with the exception of one animal), and the likelihood of infection with FeMV might therefore have been higher. Another aspect that may explain the higher FeMV seroprevalence in Chile than in other countries might be the different global distribution of the two FeMV genotypes, since no serological data were obtained on the American continents, although FeMV RNA has been detected in the USA [6] and Brazil [7]. A complete genome sequence was available for the US strain, only. An amino acid sequence comparison between the FeMV-1 strain used in this study and the US
Fig. 3 Comparison of seroprevalence rates of FeMV antibodies in samples taken from male and female cats in Chile. Statistical significance is shown as the $p$-value

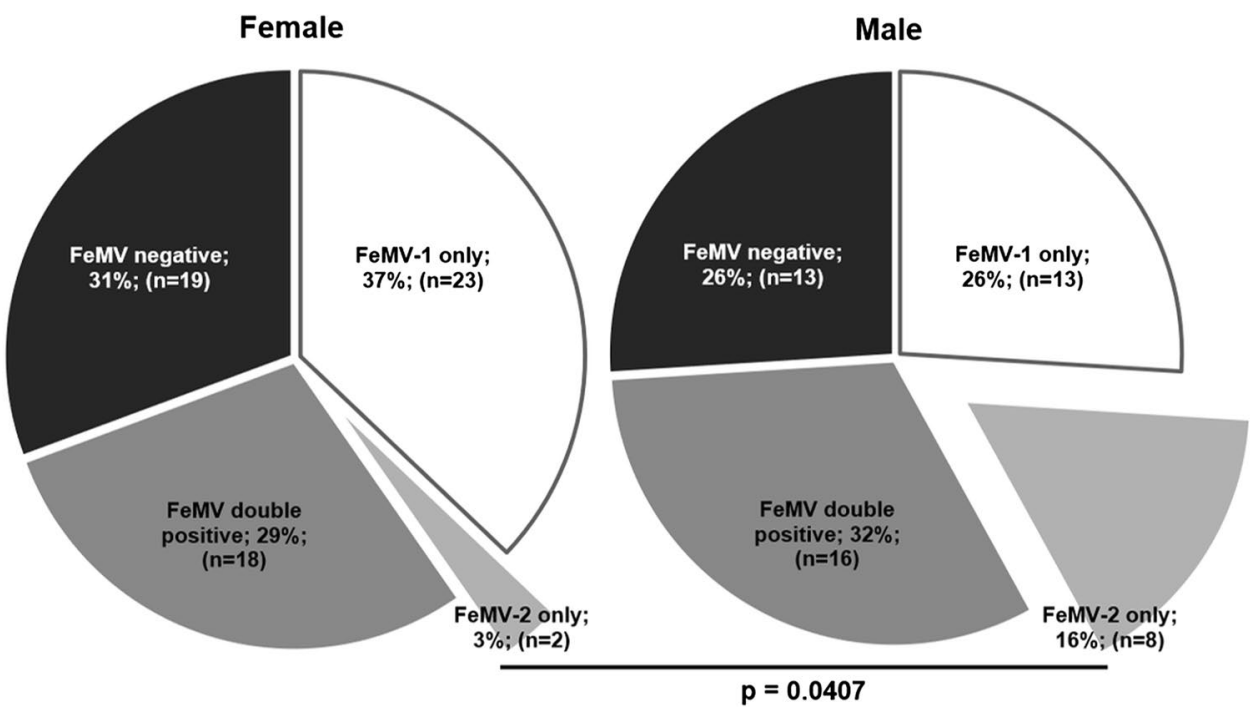

Springer 
strain (accession no. KR014147) revealed 97.11\% sequence identity in the $\mathrm{N}$ protein, $86.73 \%$ in the P protein, $96.14 \%$ in the $\mathrm{M}$ protein, $94.29 \%$ in the $\mathrm{F}$ protein, $94.79 \%$ in the $\mathrm{H}$ protein, and $96.05 \%$ in the RNA polymerase protein. These data point towards a limited variability of FeMV-1 globally, and cross-reactivity of the antibodies generated can be assumed. A high degree of similarity among all available FeMV-1 strains was also shown previously [21]. The overall amino acid sequence identity in the N, P, M, F, H and RNA polymerase protein between FeMV-1 and FeMV-2 used in this study was found to be $90.94 \%, 77.19 \%, 91.39 \%, 89.50 \%$, $86.53 \%$, and $90.64 \%$, respectively. Therefore, assays based solely on FeMV-1 sequences or proteins might underestimate the prevalence of FeMV-2. Using our IFA approach, we detected a significantly higher seroprevalence of FeMV-2 in male cats. This might be explained by the lack of neutering in this cohort, resulting in closer and more frequent social contacts between animals, increased roaming distance, urine spraying, and sexual activity [22, 23]. Like for FeMV-2, the seroprevalence of feline immunodeficiency virus (FIV) and feline leukemia virus (FeLV)' as well as 'canine distemper virus (CDV) have also been reported to be higher in male cats [24]. Since no such correlation was detected for FeMV1 , an alternative route of transmission may be considered.

Correlations with FeLV, FIV and ??canine distemper virus?? (CDV) data evaluated based on the samples of this investigation in the course of a previous study [25] showed that two cats that were double positive for FeMV-1 and FeMV-2 were also positive for FIV antibodies. All but one of the animals were negative for CDV antibodies [25]. Out of ten animals that were positive for FeLV antigen, two were positive for FeMV-1 only, two positive for were FeMV-2 only, and two were positive for both FeMV-1 and FeMV-2. Furthermore, four FeLV-antigen-positive cats were FeMV negative. Due to the limited sample size, the statistical correlation between FIV, CDV and FeLV status of cats in comparison to FeMV antibodies could not be determined. It is, however, important to note that FeMV results obtained using the IFA used in the present study are not attributable to crossreactivity against either of those feline viruses, especially as might be expected in the case of CDV. The serum samples that were analyzed were collected between 2008 and 2010 and between 2015 and 2016. No differences regarding the seroprevalence of FeMV antibodies were detected between these two periods. It can be concluded that the virus was circulating as early as 2008 in Chile, which is similar to what was reported by Woo et al. in Hong Kong [2].

In conclusion, our findings demonstrate a high seroprevalence of FeMV in Chilean free-roaming cats. Our IFA data indicate that FeMV seroprevalence data might be higher than reported in previous serological surveys based solely on the antibody response against a single viral protein. The susceptibility of female and male animals to both FeMV genotypes should be investigated further, as our data suggest possible sex-specific effects regarding the seroprevalence of different FeMV genotypes.

Acknowledgements We gratefully acknowledge the local inhabitants of rural communities for kindly giving us the opportunity to sample their domestic cats. We are grateful to CONAF, especially Patricio Contreras, Patricia Barría, Andrea Bahamonde and Dennis Aldrigde; SAG, especially to Diego Ramírez and Rodrigo Villalobos; the Ministry of the Environment, especially to Sandra Díaz; Tantauco Park, especially to Alan Bannister and Catherine Chirgwin; and the Valdivian Coastal Reserve for logistic support. Special thanks to Debora Mera, Diego Peñaloza, Gonzalo Canto, Camila Núñez, for their valuable support in sample collection.

Author contributions JB performed the experiments, analyzed data, wrote the first draft of the manuscript, and designed the study; IS collected samples, provided data regarding the animals, and reviewed the manuscript; AC collected samples, provided data regarding the animals, and reviewed the manuscript, JM collected samples, provided data regarding the animals, and reviewed the manuscript; TWV supervised the study and reviewed the manuscript; $\mathrm{CN}$ supervised the study, organized sample collection, provided data regarding the animals, and reviewed the manuscript; and MS supervised the experiments, designed the study, and reviewed the manuscript.

Funding Open Access funding enabled and organized by Projekt DEAL. This work was supported by Boehringer Ingelheim Veterinary Research Center GmbH \& Co. KG, Hannover, Germany, CONICYT FONDECYT Iniciación 11150934 (CN), Morris Animal Foundation D15ZO-413 (CN), National Geographic Society C309-15 (CN), Mohamed bin Zayed Species Conservation Fund 152510351 (CN), ANID PAI 77190064 (CN), Wild Felid Association (IS), Fondo Interno UNAB N ${ }^{\circ}$ DI-778-15/R (JM), Morris Animal Foundation no. D16Z825 (JM), CONICYT FONDECYT Regular no. 1161593 (JM, CN), Fondecyt-Regular 1151693.

\section{Compliance with ethical standards}

Conflict of interest The authors declare that they have no conflict of interest. The founding sponsors had no role in the design of the study, in the collection, analysis, or interpretation of data, in the writing of the manuscript, or in the decision to publish the results.

Ethical approval The collection of samples was performed with the informed consent of the owners and under considerations of animal welfare and ethical aspects under the approval of Animal Ethics Committee of the Institute of Ecology and Biodiversity at the Universidad de Chile, resolution of 20 November 2015. Sera were imported to Germany under permission number 24-9152.81 (EFG-Nr. 38/2016) provided by the Saxon State Ministry for Social and Consumer Protection.

Open Access This article is licensed under a Creative Commons Attribution 4.0 International License, which permits use, sharing, adaptation, distribution and reproduction in any medium or format, as long as you give appropriate credit to the original author(s) and the source, provide a link to the Creative Commons licence, and indicate if changes were made. The images or other third party material in this article are included in the article's Creative Commons licence, unless indicated otherwise in a credit line to the material. If material is not included in the article's Creative Commons licence and your intended use is not permitted by statutory regulation or exceeds the permitted use, you will 
need to obtain permission directly from the copyright holder. To view a copy of this licence, visit http://creativecommons.org/licenses/by/4.0/.

\section{References}

1. Rima B, Balkema-Buschmann A, Dundon WG et al (2019) ICTV virus taxonomy profile: paramyxoviridae. J Gen Virol 100:1593-1594

2. Woo PCY, Lau SKP, Wong BHL et al (2012) Feline morbillivirus, a previously undescribed paramyxovirus associated with tubulointerstitial nephritis in domestic cats. Proc Natl Acad Sci USA 109:5435-5440

3. Sakaguchi S, Nakagawa S, Yoshikawa R et al (2014) Genetic diversity of feline morbilliviruses isolated in Japan. J Gen Virol 95:1464-1468

4. Sieg M, Heenemann K, Ruckner A et al (2015) Discovery of new feline paramyxoviruses in domestic cats with chronic kidney disease. Virus Genes 51:294-297

5. Lorusso A, Di Tommaso M, Di Felice E et al (2015) First report of feline morbillivirus in Europe. Vet Ital 51:235-237

6. Sharp CR, Nambulli S, Acciardo AS et al (2016) Chronic infection of domestic cats with feline morbillivirus, United States. Emerg Infect Dis 22:760-762

7. Darold GM, Alfieri AA, Muraro LS et al (2017) First report of feline morbillivirus in South America. Arch Virol 162:469-475

8. Yilmaz H, Tekelioglu BK, Gurel A et al (2017) Frequency, clinicopathological features and phylogenetic analysis of feline morbillivirus in cats in Istanbul, Turkey. J Feline Med Surg 19:1206-1214

9. McCallum KE, Stubbs S, Hope N et al (2018) Detection and seroprevalence of morbillivirus and other paramyxoviruses in geriatric cats with and without evidence of azotemic chronic kidney disease. J Vet Intern Med 32:1100-1108

10. Mohd Isa NH, Selvarajah GT, Khor KH et al (2019) Molecular detection and characterisation of feline morbillivirus in domestic cats in Malaysia. Vet Microbiol 236:108382

11. Ou J, Ye S, Xu H et al (2020) First report of feline morbillivirus in mainland China. Arch Virol 165:1837-1841

12. Park E-S, Suzuki M, Kimura M et al (2016) Epidemiological and pathological study of feline morbillivirus infection in domestic cats in Japan. BMC Vet Res 12:228

13. de Luca E, Crisi PE, Marcacci M et al (2020) Epidemiology, pathological aspects and genome heterogeneity of feline morbillivirus in Italy. Vet Microbiol 240:108484
14. Sieg M, Busch J, Eschke M et al (2019) A new genotype of feline morbillivirus infects primary cells of the lung, kidney, brain and peripheral blood. Viruses 11:146

15. Sutummaporn K, Suzuki K, Machida N et al (2019) Association of feline morbillivirus infection with defined pathological changes in cat kidney tissues. Vet Microbiol 228:12-19

16. Bartlett PC, van Buren JW, Neterer M et al (2010) Disease surveillance and referral bias in the veterinary medical database. Prev Vet Med 94:264-271. https://doi.org/10.1016/j.preve tmed.2010.01.007

17. Marino CL, Lascelles BDX, Vaden SL et al (2014) Prevalence and classification of chronic kidney disease in cats randomly selected from four age groups and in cats recruited for degenerative joint disease studies. J Feline Med Surg 16:465-472. https:// doi.org/10.1177/1098612X13511446

18. Arikawa K, Wachi A, Imura Y et al (2017) Development of an ELISA for serological detection of feline morbillivirus infection. Arch Virol 162:2421-2425

19. Sieg M, Vahlenkamp A, Baums CG et al (2018) First complete genome sequence of a feline morbillivirus isolate from germany. Genome Announc 6:e00244-e318

20. QuickCalcs Web site. https://www.graphpad.com/quickcalcs/conti ngency2/. Accessed 13 May 2020

21. Stranieri A, Lauzi S, Dallari A et al (2019) Feline morbillivirus in Northern Italy: prevalence in urine and kidneys with and without renal disease. Vet Microbiol 233:133-139

22. Gunther I, Raz T, Klement E (2018) Association of neutering with health and welfare of urban free-roaming cat population in Israel, during 2012-2014. Prev Vet Med 157:26-33

23. Cafazzo S, Bonanni R, Natoli E (2019) Neutering effects on social behaviour of urban unowned free-roaming domestic cats. Animals (Basel) 9:1105

24. Bande F, Arshad SS, Hassan L et al (2012) Prevalence and risk factors of feline leukaemia virus and feline immunodeficiency virus in peninsular Malaysia. BMC Vet Res 8:33

25. Sacristan I, Sieg M, Acuna F et al (2019) Molecular and serological survey of carnivore pathogens in free-roaming domestic cats of rural communities in southern Chile. J Vet Med Sci 81:1740-1748

Publisher's Note Springer Nature remains neutral with regard to jurisdictional claims in published maps and institutional affiliations. 